\title{
Accretion Disks in the SW Sex Stars
}

\author{
D. W. Hoard and Paula Szkody \\ Department of Astronomy, University of Washington, Box 351580, \\ Seattle WA 98195 USA
}

\begin{abstract}
We present recent results of time-resolved optical spectroscopy on two SW Sex stars, PG 0859+415 and BH Lyncis.
\end{abstract}

\section{Observations}

We have obtained medium resolution $(2 \AA)$ optical spectra of the SW Sex stars PG 0859+415 (on 1995 Mar 5 UT) and BH Lyn (on 1994 Nov 18 UT, 1995 Jan 21 UT, and 1995 Nov 23 UT) using the Double Imaging Spectrograph (DIS) on the Apache Point Observatory (APO) $3.5 \mathrm{~m}$ telescope. In addition, we obtained CCD photometry of BH Lyn over $>1$ orbit on 1995 Oct 22 UT with the $0.76-\mathrm{m}$ telescope at Manastash Ridge Observatory (MRO).

\section{2. $P G 0859+415$}

The history of PG $0859+415$ and our recent results are presented in depth in Hoard \& Szkody (1996), so are not reiterated in detail here. Our primary conclusion is that its peculiar observational features can be explained by an enhancement to the disk thickness near the accretion stream impact site.

\section{BH Lyncis}

BH Lyn has also been studied by Thorstensen et al. (1991) and Dhillon et al. (1992). We have derived an updated ephemeris using 19 eclipse timings (this work, Andronov et al. 1989, Dhillon et al. 1992, Chen et al. 1990):

$$
\mathrm{HJD}(\min )=2447180.3366(2)+0.15587535(6) E .
$$

Our Balmer tomograms of BH Lyn (see Fig. 1) are similar to the $\mathrm{H} \alpha$ tomogram of the SW Sex star PX And obtained by Hellier \& Robinson (1994). They propose a model in which the accretion stream continues over the disk after its initial impact at the outer edge, and re-impacts at a secondary site in the inner disk. The absorption feature that appears in the lines of the SW Sex stars is then due to absorption of the underlying disk emission by the overflowing accretion stream. While it remains to be seen whether this explanation is completely satisfying, some form of disk overflow does seem to be at work in BH Lyn; however, it appears to be an unstable process that produces significant changes over time in the observational characteristics of the system. For example, when 


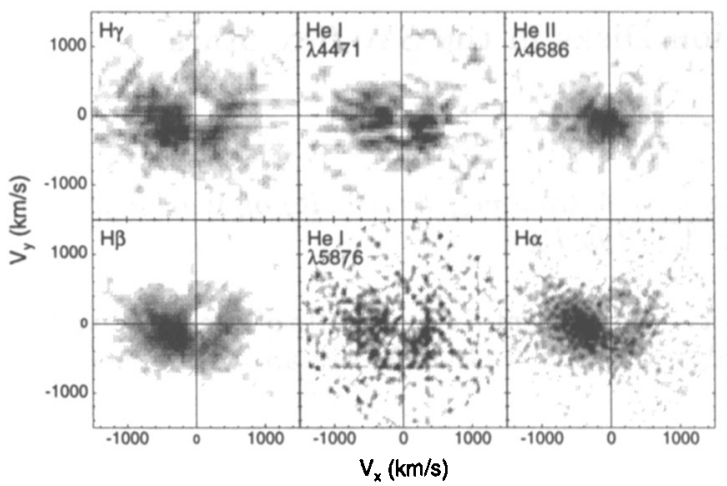

Figure 1. Doppler tomograms of BH Lyn.
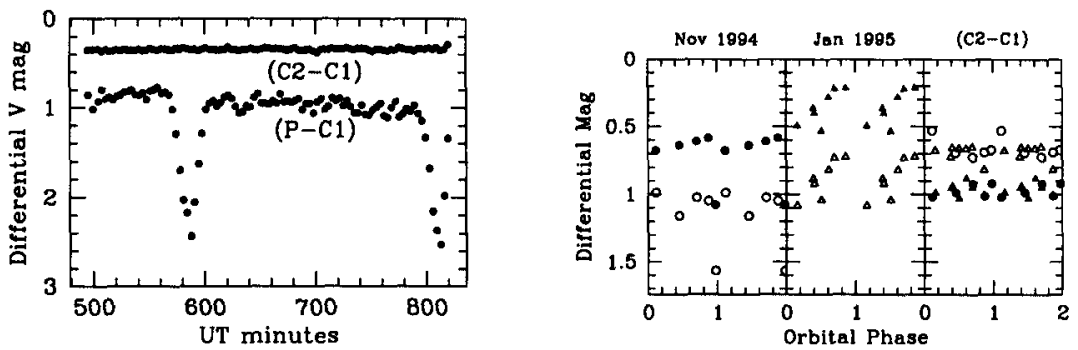

Figure 2. CCD light curves of BH Lyn. (left) From MRO: the deep eclipse and lack of a pre-eclipse hump are apparent. (right) From APO (open $=$ red, filled $=$ blue): phase coverage is sparse because the images were only taken to check slit positioning, but a pre-eclipse hump is seen in the Jan light curve.

the light curve (see Fig. 2) displays a pre-eclipse hump, it may indicate that the accretion rate has increased so that material is "piling up" in the disk regions predicted by Hirose et al. (1991) and thereby producing the enhanced absorption around $\phi \approx 0.5$.

\section{References}

Andronov, I. L., Kimeridze, G. N., Richter, G. A., and Smykov, V. P. 1989, IBVS, no.3388

Chen, J.-S., Wei, M.-Z., \& Liu, X.-W. 1990, Acta Astron. Sin., 31, 305

Dhillon, V. S., Jones, D. H. P., Marsh, T. R., and Smith, R. C. 1992, MNRAS, 258,225

Hellier, C. \& Robinson, E. L. 1994, ApJ, 431, L107

Hirose, M., Osaki, Y., \& Mineshige, S. 1991, PASJ, 43, 809

Hoard, D. W. \& Szkody, P. 1996, ApJ, accepted (to appear in October 1996)

Thorstensen, J. R., Davis, M. K., \& Ringwald, F. A. 1991, AJ, 102, 683 